\title{
INCENTIVO AO PRÉ- NATAL DO PARCEIRO NA ATENÇÃO PRIMÁRIA EM SAÚDE: UM RELATO DE EXPERIÊNCIA
}

\author{
ENCOURAGEMENT OF PARTNER PRENATAL IN PRIMARY HEALTH \\ CARE: AN EXPERIENCE REPORT
}

Ana Emília Araújo de Oliveira
Gizele Marinho de Farias

RESUMO: INTRODUÇÃO: A Política Nacional de Atenção Integral à Saúde do Homem (PNAISH), instituída pelo Ministério da Saúde em 2009 tem como objetivo ampliar e facilitar o acesso da população masculina às ações e aos serviços de assistência integral à saúde no Sistema Único de Saúde (SUS); aposta na perspectiva de inclusão do tema paternidade e cuidado, por meio do Pré-Natal do Parceiro, que trata da ampliação do acesso e acolhimento dos homens aos serviços e programas de saúde, incentivando o envolvimento integral dos homens na gestação, no parto, no cuidado e na educação das crianças, aprimorando os vínculos familiares. OBJETIVO: Sensibilizar a equipe de saúde, as gestantes e seus companheiros sobre a importância da realização do pré-natal do parceiro. MÉODOS: Estudo descritivo, tipo relato de experiência, elaborado no contexto do atendimento durante o Pré- Natal na UBS Adriana Bezerra no Município de Campina Grande- PB no mês de Setembro de 2018. RESULTADOS: Alcançar o maior número de homens acompanhando as suas parceiras na consulta de pré - natal e realizando o Pré-Natal do Parceiro. CONCLUSÃO: Conhecimento sobre o Pré-Natal do parceiro e a adesão do homem ao serviço de saúde.

Palavras chave: Saúde do Homem; Pré-Natal; Serviços de Saúde.

ABSTRACT :INTRODUCTION: The National Policy for Integral Attention to Men's Health (PNAISH), instituted by the Ministry of Health in 2009, aims to expand and facilitate the access of the male population to actions and services of comprehensive health care in the Unified Health System ( SUS); bet on the perspective of inclusion of the theme paternity and care, through the Prenatal Partner, which deals with the expansion of access and acceptance of men to health services and programs, encouraging the full involvement of men in pregnancy, childbirth, care and education of children, improving family ties. OBJECTIVE: Sensitize the health team, pregnant women and their partners about the importance of performing prenatal partner. METHODOLOGY: Descriptive study, type experience report, elaborated in the 
context of prenatal care at UBS Adriana Bezerra in the city of Campina Grande-PB in September 2018. Results: Reaching the largest number of men accompanying their partners in the prenatal consultation and performing the Prenatal Partner. CONCLUSION: Knowledge about the prenatal partner and the adhesion of men to health services.

Keywords: Men's Health; Prenatal; Health Services. 


\section{INTRODUÇÃO}

A Atenção Primária em Saúde (APS) como porta de entrada do sistema de saúde brasileiro, teve um avanço consideravelmente desde a efetivação da Estratégia Saúde da Família (ESF), seja ampliando o acesso da população aos serviços, seja disponibilizando mais ações de promoção, prevenção e tratamento (BRASIL,2011).

Sendo a ESF o primeiro contato da gestante com o serviço público de saúde, esta unidade assistencial tem como competências acolher a mulher na suspeita de gravidez ou que deseja engravidar, solicitar os primeiros exames pré-natais, incluindo USG do primeiro trimestre, iniciar suplemento vitamínico para gestante, avaliar situação vacinal, investigar estado psicossocial das gestantes, acompanhar o pré-natal da mulher até o parto e, articular com outros setores para referência e contra referências necessárias.

A gestação é um momento especial repleto de significados e emoções para a família e um acontecimento que demanda uma série de ações pela área da saúde. Nos últimos anos, um tema tem emergido cada vez com mais força, exigindo debates, ações e principalmente, uma mudança de olhar por parte dos/as pesquisadores/as, gestores(as), trabalhadores/as de saúde e ativistas: a importância do envolvimento consciente e ativo do pai/parceiro (BRASIL, 2017).

Usualmente, a gestação é definida pelas mudanças observadas no corpo feminino a partir dos meses iniciais. Nesse contexto, muitas vezes a paternidade parece só existir quando a criança nasce ou mesmo quando ela já está mais crescida. Muitos homens, de diferentes idades, demonstram desejo de participar ou efetivamente participam em todos os momentos da gravidez, desde a decisão compartilhada de ter um filho, passando por todas as fases da gestação, até o desenvolvimento da criança (UNFPA e Instituto PAPAI, 2007).

Em busca de ações que poderiam aumentar a busca do atendimento primário com relação ao homem, o Ministério da Saúde lançou a Política Nacional de Atenção Integral à Saúde do Homem (PNAISH) instituída pela Portaria GM/MS nº 1.944, de 27 
de agosto de 2009, esta tem como objetivo facilitar e ampliar o acesso com qualidade da população masculina, na faixa etária de 20 a 59 anos, às ações e aos serviços de assistência integral à saúde da Rede SUS, mediante a atuação nos aspectos socioculturais, sob a perspectiva relacional de gênero e na lógica da concepção de linhas de cuidado que respeitem a integralidade da atenção, contribuindo de modo efetivo para a redução da morbimortalidade e melhores condições de saúde da população. A PNAISH aposta na perspectiva da inclusão do tema da paternidade e cuidado, por meio do Pré-Natal do Parceiro, nos debates e nas ações voltadas para o planejamento reprodutivo como uma estratégia essencial para qualificar a atenção à gestação, ao parto e ao nascimento, estreitando a relação entre trabalhadores de saúde, comunidade e, sobretudo, aprimorando os vínculos afetivos familiares dos usuários e das usuárias nos serviços ofertados (MS, 2013).

Segundo Ministério da Saúde, Brasil (2009), os homens têm dificuldades de reconhecer suas necessidades, cultivando o pensamento mágico que rejeita a possibilidade de adoecer. Uma possível solução desta problemática não está ligada em aumentar os números de profissionais de saúde do sexo masculino, mas, em reconstruir as atitudes desses profissionais, independente do sexo em frente à atenção às demandas de saúde do homem (SILVA, 2013p. 14).

No atendimento às gestantes durante a consulta de pré-natal no mês de Setembro de 2018 realizado na UBS Adriana Bezerra, equipe Santa Rosa I, foi observado a não adesão do homem ao Pré-natal, dessa forma, o relato de experiência propõe sensibilizar a equipe de saúde, as gestantes e seus companheiros sobre a importância da realização do pré-natal do parceiro e diante disso, alcançar o maior número de homens acompanhando as suas parceiras na consulta de pré - natal. 


\section{REFERENCIAL TEÓRICO}

A Rede Cegonha, lançada em 2011, consiste numa rede de cuidados que visa assegurar à mulher o direito ao planejamento reprodutivo e à atenção humanizada à gravidez, ao parto e ao puerpério, bem como o direito ao nascimento seguro e ao crescimento e desenvolvimento saudáveis das crianças. Esta Rede visa proporcionar às mulheres saúde, qualidade de vida e bem-estar durante a gestação, o parto, o pós-parto e o desenvolvimento da criança até os dois primeiros anos de vida. Ainda tem a finalidade de reduzir a mortalidade materna e infantil e garantir os direitos sexuais e reprodutivos de mulheres e de homens, de jovens e de adolescentes. .(MS, 2016).

A proposta qualifica os serviços ofertados pelo Sistema Único de Saúde (SUS) no planejamento reprodutivo, na confirmação da gravidez, no pré-natal, parto e puerpério, constituindo uma oportunidade propícia para a inclusão e participação ativa dos pais/parceiros. Além disso, a Rede Cegonha sistematiza e institucionaliza um modelo de atenção ao parto e ao nascimento que vem sendo discutido e construído no país desde 1990 com base no pioneirismo e na experiência de médicos, enfermeiros, parteiras, doulas, acadêmicos, antropólogos, sociólogos, gestores, formuladores de políticas públicas, gestantes, ativistas e instituições de saúde, entre outros. (MS, 2016).

Segundo Ministério da Saúde, Brasil (2009), os homens têm dificuldades de reconhecer suas necessidades, cultivando o pensamento mágico que rejeita a possibilidade de adoecer. Uma possível solução desta problemática não está ligada em aumentar os números de profissionais de saúde do sexo masculino, mas, em reconstruir as atitudes desses profissionais, independente do sexo em frente à atenção às demandas de saúde do homem (SILVA, 2013p. 14).

Figueiredo (2005) retrata algumas suposições da baixa procura dos homens nos serviços de atenção primária, podendo estar ligada as questões culturais em que o homem frequentar a Unidade Básica de Saúde ou qualquer outro serviço de saúde de níveis de complexidade os tornariam feminizados e fragilizados já que o 
local é mais frequentado, principalmente, por mulheres e composto de equipes de profissionais, formada em sua maioria, também por mulheres.

Em busca de ações que poderiam aumentar a busca do atendimento primário, o Ministério da Saúde mediante a Política Nacional de Atenção Integral à Saúde do Homem (PNAISH) instituída pela Portaria GM/MS n 1.944, de 27 de agosto de 2009, que tem como objetivo facilitar e ampliar o acesso com qualidade da população masculina às ações e aos serviços de assistência integral à saúde da Rede SUS, mediante a atuação nos aspectos socioculturais. A PNAISH aposta na perspectiva da inclusão do tema da paternidade e cuidado, por meio do Pré- Natal do Parceiro, nos debates e nas ações voltadas para o planejamento reprodutivo como uma estratégia essencial para qualificar a atenção à gestação, ao parto e ao nascimento, estreitando a relação entre trabalhadores de saúde, comunidade e, sobretudo, aprimorando os vínculos afetivos familiares dos usuários e das usuárias nos serviços ofertados (MS, 2013).

\section{METODOLOGIA}

Trata-se de um estudo descritivo, tipo relato de experiência, elaborado no contexto do atendimento durante o Pré- Natal na UBS Adriana Bezerra no Município de Campina Grande- PB no mês de Setembro de 2018.

O relato de experiência apresenta reflexão sobre um conjunto de ações que abordam uma situação vivenciada no âmbito profissional de interesse a comunidade científica, sendo assim, uma importante ferramenta da pesquisa descritiva. (CAVALCANTE E LIMA, 2012).

Por se tratar de um relato de experiência, tornou-se dispensável a avaliação deste estudo pelo Comitê de Ética contudo, foi mantido o respeito e anonimato dos participantes em concordância a resolução 466/12 aprovada pelo Conselho Nacional de Saúde.

O Município de Campina Grande - PB tem uma população de 385.276 habitantes e uma área territorial de $621 \mathrm{~km}^{2}$. É considerado o segundo mais 
populoso do estado da Paraíba, ficando a 120 km da capital do estado, João Pessoa (IBGE, 2010).

A rede municipal de saúde é formada por um conjunto de instituições públicas, privadas e filantrópicas, que desenvolve ações assistenciais desde o nível básico até o nível terciário. A Secretaria Municipal de Saúde trabalha com divisão territorial cujos serviços de saúde estão organizados em dez distritos sanitários (DS), com 108 Equipes de Saúde. A Unidade Básica de Saúde Adriana Bezerra localizase no Bairro de Santa Rosa, onde faz parte do Distrito Sanitário ( DS) X, atendendo o nível primário em Saúde.

\section{RELATO DE EXPERIÊNCIA}

O relato refere-se ao atendimento realizado pela Docente do Curso de Enfermagem da Universidade Estadual da Paraíba - UEPB, juntamente com os discentes do $8^{\circ}$ Período de Enfermagem, durante o atendimento de Pré-Natal. Foi realizado no turno da tarde do mês de Setembro de 2018, com agenda aberta para marcação e atendimento as gestantes para realização da Consulta de Enfermagem.

Durante o atendimento, foi observada a não adesão do homem ao Pré- Natal juntamente com a parceira. Das 20 (vinte) gestantes atendidas, apenas uma ( 01) estava sendo acompanhada pelo parceiro e durante a consulta, nos informou saber e explicar sobre a existência do Pré-Natal do Homem.

Com o objetivo de sensibilizar a equipe de saúde, as gestantes e seus companheiros sobre a importância da realização do pré-natal do parceiro e diante disso, alcançar o maior número de homens acompanhando as suas parceiras na consulta de pré - natal, foi proposto em cada consulta, o diálogo sobre a temática em questão, explicando o objetivo da Política Nacional de Atenção Integral à Saúde do Homem (PNAISH) e mostrando na "caderneta da gestante", (Figura 1.) e em anexo à página 48, a parte que orienta sobre o atendimento ao homem, no Pré-Natal do Parceiro. (Figura 2.) 
Com relação à Equipe de Saúde, foi realizada uma reunião composta por ACS, médico e Técnico de Enfermagem da Equipe Santa Rosa I, onde foi dialogado sobre a importância da Política Nacional de Atenção Integral à Saúde do Homem (PNAISH), no intuito de sensibilizar a equipe para a busca ativa e orientação sobre a importância da participação do homem no Pré- Natal e também foi impresso "folder" explicativo do Ministério da Saúde, sobre a Saúde e o acesso do Homem nos Serviços ( Figura 3.)

Figura 1. Capa da Caderneta da Gestante.

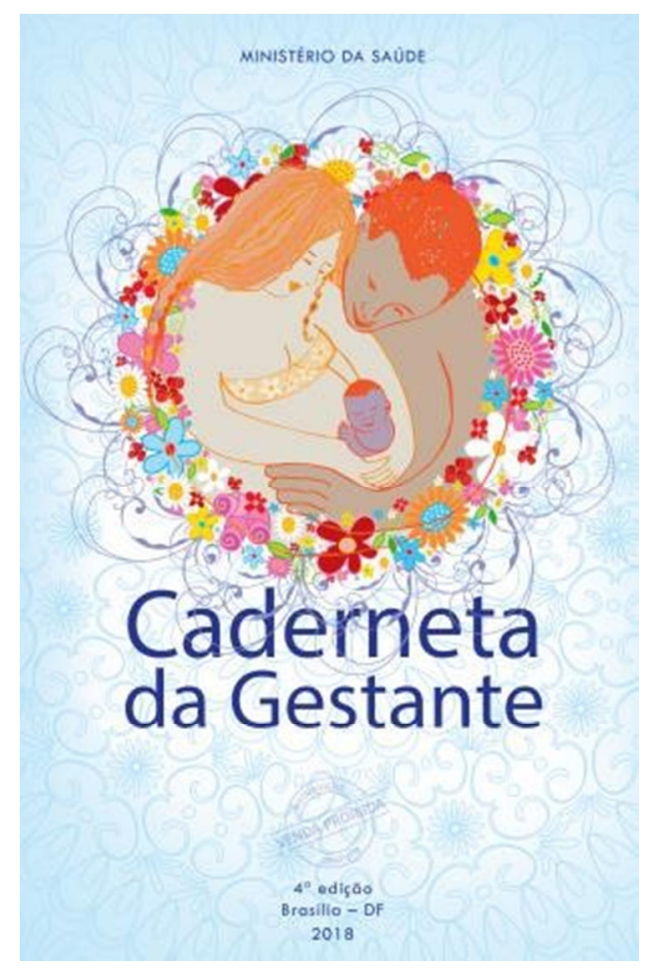


Figura 2. Anexo à página 48 da Caderneta da Gestante:o Pré-Natal do Parceiro.

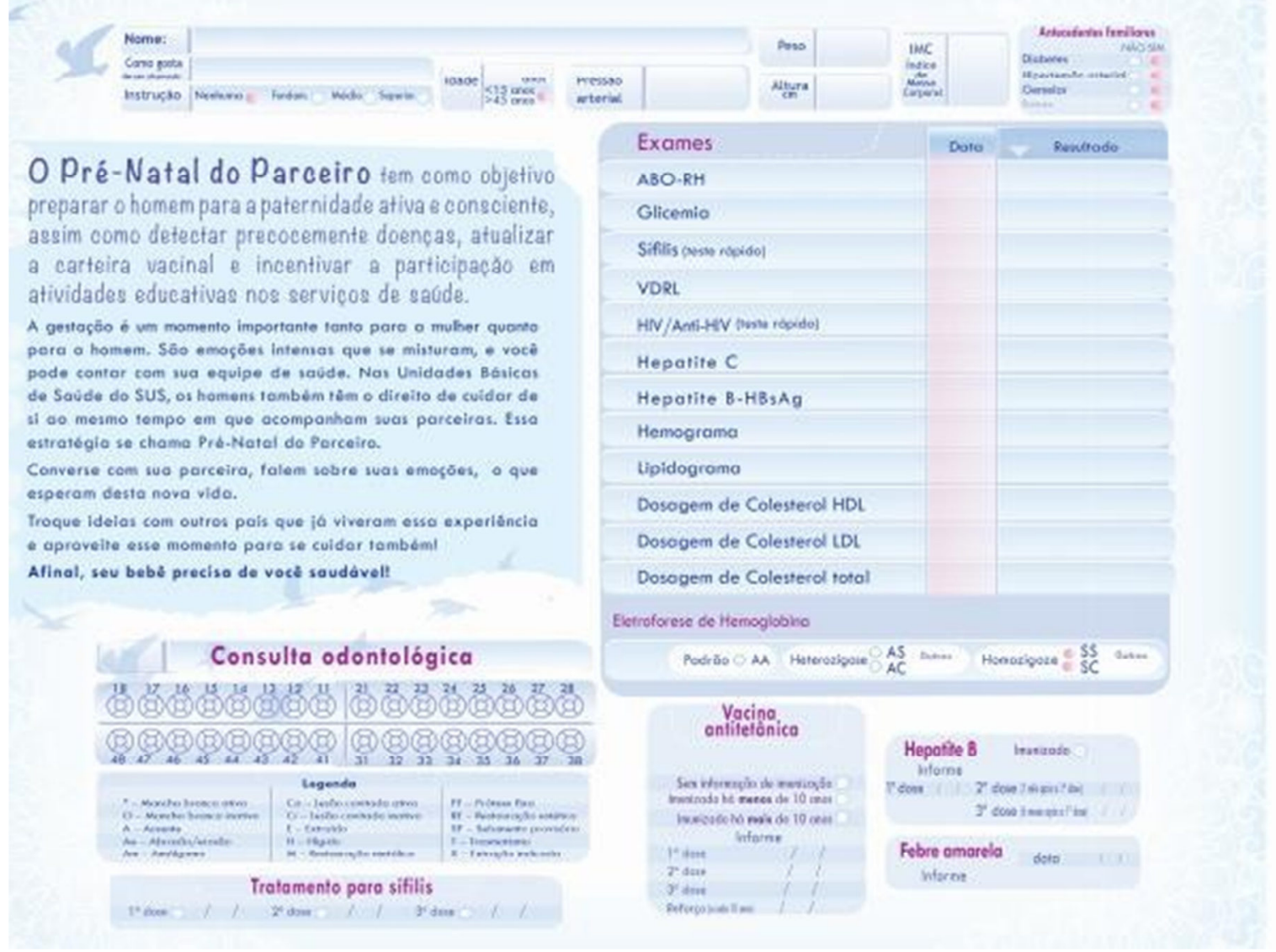


Figura 3. Folder do Ministério da Saúde sobre a Saúde do Homem.

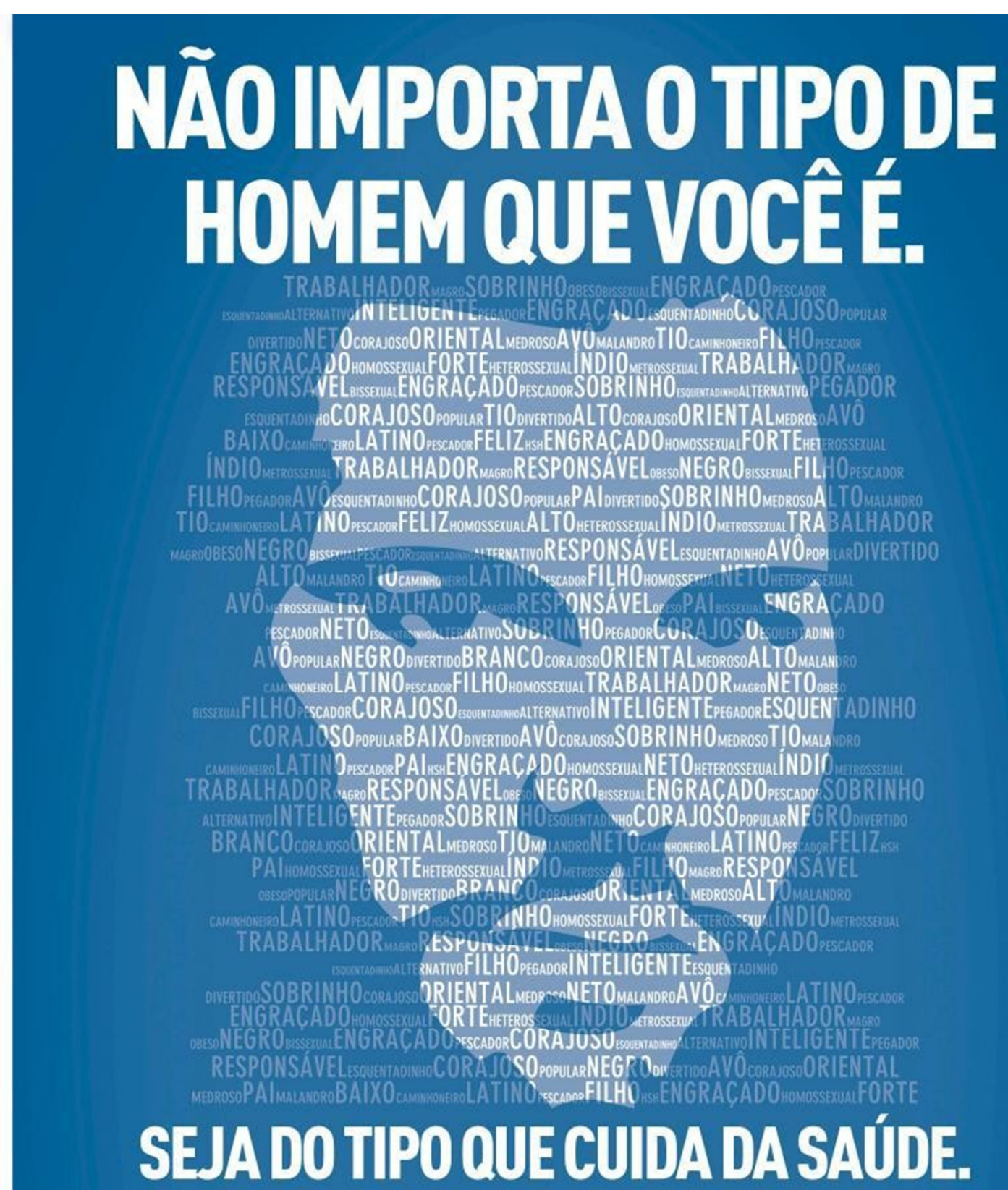

A Política Nacional de Atenção Integral à Saúde do Homem promove ações específicas para você CUIDAR de si e das pessoas que você quer bem. Este é um direito seu. Prevenção é qualidade de vida. Homem, escolha Saúde! PROCURE UMA UNIDADE DE SAÚdE. 


\section{REFERÊNCIAS BIBLIOGRÁFICAS}

BRASIL. Ministério da Saúde. Política Nacional de Atenção Integral à Saúde do Homem. Brasília, DF: Ministério da Saúde, 2011.

BRASIL. Ministério da Saúde. Portaria no 1.459, de 24 de junho de 2011. Institui no âmbito do Sistema Único de Saúde a Rede Cegonha. 2017. Disponível em http://bvsms.saude.gov.br/bvs/saudelegis/gm/2011/prt1459_24_06_2011.html> acesso 19 outubro de 2019.

CAVALCANTE, B. L. L.; LIMA U. T. S. Relato de experiência de uma estudante de Enfermagem.. J Nurs Health, Pelotas (RS) 2012 jan/jun; 1 (2): 94-103.. Acesso em Outubro de 2019.

IBGE- INSTITUTO BRASILEIRO DE GEOGRAFIA E ESTATÍSTICA, 2010. Pesquisa População Nacional Campina Grande, 2010.

INSTITUTO PAPAI (2007) UNFPA Relações compartilhadas. Cartilha. Apoio: CNB-CUT. Acesso em 11/11/2019.

Silva, M. M. J. S.; Cardoso, E. P.; Calheiros, C. A. P.; Rodrigues, E. O. M. A.; Leite, E. P. R.A \& Rocha, L. C. D. (2013). O envolvimento paterno na gestação sob o olhar de gênero. Rev enferm UFPE on line, 7(5), p. 14. 